\title{
Variabilidade espacial das frações granulométricas e da produção de bananeira 'Prata Anã'
}

\author{
Spatial variability of the granulometric fractions \\ and the production of banana cultivar 'Prata Anã' \\ Moises Zucoloto ${ }^{1 *}$, Julião Soares de Souza Lima ${ }^{2}$, \\ Ruimario Inacio Coelho ${ }^{3}$, Wayson Zancanella Quartezani ${ }^{4}$
}

RESUMO

O presente trabalho teve como objetivo estudar a variabilidade espacial das frações granulométricas e a produção de uma parcela experimental cultivada com bananeira 'Prata Anã', por meio de técnicas de análises exploratórias e geoestatística. As amostras de solo foram coletadas na região de projeção da copa da cultura na profundidade de 0-0,2 m, utilizando uma malha regular, totalizando 100 pontos amostrais, espaçados $6 \times 4 \mathrm{~m}$. Em cada amostra foram determinadas as frações de areia grossa (AG), areia fina (AF), areia total (AT), silte (Sil) e argila (AR) e a produção de cada planta. A massa do cacho (MC) não apresentou correlação significativa com nenhuma das frações granulométricas.

Palavras-chave: Musa ssp., dependência espacial, geoestatística, krigagem.

\section{ABSTRACT}

The objective was to study the spatial variability of the particle size fraction and the production of an experimental parcel cultivated with banana cultivar 'Prata Anã', through techniques of exploratory analysis and geostatistics. The data were collected in the area under the crown projection of the plants at a depth of 0-0.2 $\mathrm{m}$, using a regular grid of $6 \times 4 \mathrm{~m}$, with a total of 100 sampling points. In each sample were determined the fractions fine sand (AF), thick sand (AG), total sand (AT), silte (Sil), clay $(A R)$ and the production of each plant. The mass of the fruit cluster $(M C)$ did not present significant correlation with any of the particle size fractions.

Key words: Musa spp., spatial dependence, geostatistics, kriging.

\section{Introdução}

A bananeira é uma planta com elevado e constante consumo de água, aumentando sua exigência no período de diferenciação floral e no início da frutificação. A disponibilidade adequada de oxigênio e a água, aliado a um solo profundo e descompactado é de fundamental importância para o bom desenvolvimento do sistema radicular da bananeira (Cordeiro, 2000).

Neste sentido, Lima et al. (2007) afirmam que a variabilidade espacial dos atributos do solo deve ser bem conhecida, a fim de minimizar os erros de amostragem e auxiliar no manejo do solo. Souza (1992) relata que a obtenção dessas informações a respeito da variabilidade espacial dos atributos do solo é de grande importância para a avaliação da fertilidade; levantamento, mapeamento e classificação de solos; desenvolvimento de esquemas mais adequados de amostragem, entre outros, visando à melhoria das condições de manejo e o incremento de produtividade das culturas.

O cultivo da bananeira apresenta um vasto campo para pesquisa em que a agricultura de precisão possa ser aplicada, perante a importância da cultura para nosso país pelo alto grau tecnológico que se tem observado em muitas lavouras e seu valor econômico. No entanto, o número de pesquisas com agricultura de precisão e a caracterização da variabilidade espacial para cultura da bananeira é pequena.

\footnotetext{
Eng $^{\circ}$ Agrônomo, Mestre em Produção Vegetal e Doutorando em Fitotecnia. Departamento de Fitotecnia/Programa de Pósgraduação-Campus Universitário CEP: 36570 000-Viçosa-MG. E-mail: moiseszucoloto@ hotmail.com

2 Prof. Associado I, Depto de Eng ${ }^{a}$ Rural, UFES, Alegre-ES. CEP: 29.500-000. E-mail: limajss@ yahoo.com.br

3 Prof. Associado I, Depto de Prod. Vegetal, UFES, Alegre-ES. CEP: 29.500-000. E-mail: rui_mario@uol.com.br

$4 \quad$ Eng $^{\circ}$ Agrônomo, Mestre em Produção Vegetal, UFES, Alegre-ES. CEP: 29.500-000. E-mail: waylson@yahoo.com.br
} 
Nesse contexto, este trabalho teve como objetivo estudar por meio de técnicas de análises exploratórias e geoestatística a variabilidade espacial das frações granulométricas e a produção de uma parcela experimental cultivada com bananeira 'Prata Anã'

\section{Material e Métodos}

O trabalho foi conduzido em uma lavoura comercial de bananeira localizada no norte do Estado do Espírito Santo, Brasil, cujas coordenadas geográficas são: $19^{\circ} 49^{\prime} 24$ ”' de Latitude Sul e $40^{\circ} 04$ ' 20" de Longitude Oeste de Greenwich. A altitude média da área utilizada está em torno de $30 \mathrm{~m}$ e declividade plana, menor que $1 \%$.

O clima, segundo a classificação de Köppen, é do tipo Aw, com estação seca no inverno e verão quente e chuvoso. Os dados climáticos obtidos da estação meteorológica Inc_013 coordenada pelo INCAPER e baseados numa série histórica compreendida nos últimos trinta anos, apresentam temperatura média anual de $24,8{ }^{\circ} \mathrm{C}$, com temperatura média do mês mais frio de $19,8^{\circ} \mathrm{C}$, do mês mais quente de $29,8^{\circ} \mathrm{C}$ e precipitação média anual acumulada de $1288 \mathrm{~mm}$.

A variedade utilizada é a Prata Anã, cultivada em um Argissolo Amarelo Distrófico Arênico, localizado nos depósitos dos tabuleiros costeiros da região de Aracruz, segundo Duarte (2000). A cultura da bananeira foi implantada no espaçamento de 3,0 $\times 2,0 \mathrm{~m}$, em sistema de fileira simples com manejo hídrico de irrigação por aspersão com lâmina d'água média variando de $100 \mathrm{~mm}$ mês para os meses mais quentes e $70 \mathrm{~mm}$ mês para os meses com menor evapotranspiração.

No centro da área comercial foi demarcada uma malha amostral regular de $2400 \mathrm{~m}^{2}$, totalizado 100 pontos, com amostras de solo coletadas no espaçamento de $6 \times 4 \mathrm{~m}$ na profundidade de $0-0,20 \mathrm{~m}$, na projeção da copa da bananeira. As amostras de solo foram encaminhadas para o Laboratório de Física do solo, para a determinação das frações granulométricas: areia grossa (AG), areia fina (AF), areia total (AT), silte (Sil) e argila (AR), conforme metodologia preconizada pela Embrapa (1997).

A produção da cultura foi avaliada no primeiro ciclo, determinando-se a massa do cacho por planta (MC), colhido entre os meses de janeiro a março de 2008 , após aproximadamente quatorze meses do plantio.

Inicialmente foi feita a análise do box-plot visando detectar a presença de pontos atípicos (outliers) para verificação de possíveis erros laboratoriais ou mesmo de digitação. Em caso de existência, fez-se a avaliação para uma possível substituição ou eliminação. Em seguida, foi realizada análise de correlação entre as frações granulométricas e a produção da bananeira.

Posteriormente, realizou-se a análise descritiva determinando à média; mediana; desvio-padrão; e os coeficientes de variação, de assimetria e de curtose. A hipótese de normalidade foi analisada pelo teste de Kolmogorov-Smirnov (KS) $(\mathrm{P} \leq 0,05)$.

Assumida a hipótese de estacionaridade, os dados foram submetidos à análise geoestatística no intuito de verificar a existência, e quando presente, quantificar o grau de dependência espacial das frações estudadas, por meio do ajuste do semivariograma descrito por Vieira (1997), que é definido pela seguinte Equação 1,

$$
\hat{\gamma}(h)=\frac{1}{2 N(h)} \sum_{i=1}^{N(h)}\left[Z\left(x_{i}\right)-Z\left(x_{i}+h\right)\right]^{2}
$$

em que: $\mathrm{N}(\mathrm{h})$ é o número de pares de valores medidos $\mathrm{Z}\left(\mathrm{x}_{\mathrm{i}}\right), \mathrm{Z}\left(\mathrm{x}_{\mathrm{i}}+\mathrm{h}\right)$, separados por um vetor $\mathrm{h}$; e $\mathrm{Z}\left(\mathrm{x}_{\mathrm{i}}\right)$ é a variável aleatória em estudo na i-ésima posição.

O software $\mathrm{GS}^{+}$(Robertson, 1998) utilizado aplica a metodologia dos mínimos quadrados para os ajustes dos modelos teóricos aos semivariogramas experimentais, determinando os parâmetros efeito pepita $\left(\mathrm{C}_{0}\right)$, patamar $\left(\mathrm{C}_{0}+\mathrm{C}\right)$ e o alcance de dependência espacial (a). Os critérios para ajuste e seleção do melhor modelo foram o coeficiente de determinação $\left(\mathrm{R}^{2}\right)$, a soma de quadrados de resíduos (SQR) e o coeficiente de correlação da validação cruzada. Sendo assim, o índice de dependência espacial (IDE) foi calculado pela relação $\left[\mathrm{C} /\left(\mathrm{C}_{0}+\mathrm{C}\right)\right]^{*} 100$, e classificado segundo Zimback (2001), que considera dependência espacial fraca (IDE<25\%); moderada $(25 \% \leq \mathrm{IDE} \leq 75 \%)$ e forte (IDE $>75 \%)$.

Comprovada a dependência espacial das frações granulométricas e da produção na área, utilizou-se o método de krigagem ordinária para interpolação de valores em locais não medidos em pixel de $2 \times 3 \mathrm{~m}$, com auxilio do software Surfer.

\section{Resultados e Discussão}

Na Tabela 1, observa-se distribuição assimétrica à direita para Sil, AR e MC indicando maior concentração dos dados abaixo da média e a esquerda para as frações AG, AF e AT. Com relação aos 
Tabela 1. Estatística descritiva das frações granulométricas do solo e produção da bananeira.

\begin{tabular}{|c|c|c|c|c|c|c|c|c|c|}
\hline \multirow{2}{*}{ Atributos } & \multirow[b]{2}{*}{ Média } & \multirow[b]{2}{*}{ Md } & \multirow[b]{2}{*}{$\mathrm{s}$} & \multicolumn{2}{|c|}{ Valores } & \multicolumn{3}{|c|}{ Coeficientes } & \multirow{2}{*}{$\mathrm{DN}$} \\
\hline & & & & Min. & Max. & $\mathrm{CV}(\%)$ & $\mathrm{C}_{\mathrm{s}}$ & $\mathrm{C}_{\mathrm{K}}$ & \\
\hline $\mathrm{MC}(\mathrm{kg})$ & 11,05 & 11 & 1,99 & 6,5 & 15,5 & 17,95 & 0,25 & $-0,43$ & ns \\
\hline $\mathrm{AG}(\mathrm{g} / \mathrm{kg})$ & 617,9 & 632,1 & 73,13 & 444,8 & 743,7 & 11,84 & $-0,66$ & $-0,23$ & ns \\
\hline $\mathrm{AF}(\mathrm{g} / \mathrm{kg})$ & 135,84 & 138,7 & 18,7 & 68,7 & 480,7 & 13,77 & $-0,05$ & $-0,30$ & ns \\
\hline AT $(g / k g)$ & 770,55 & 776,35 & 44,01 & 666,5 & 860,8 & 5,71 & $-0,60$ & $-0,03$ & ns \\
\hline Sil (g/kg) & 4,77 & 4,21 & 3,33 & 0,42 & 17,02 & 69,81 & 1,86 & 3,9 & $*$ \\
\hline $\mathrm{AR}(\mathrm{g} / \mathrm{kg})$ & 214,28 & 214,22 & 41,17 & 137,5 & 305,22 & 19,21 & 0,24 & $-0,29$ & ns \\
\hline
\end{tabular}

MC: massa do cacho; AG: areia grossa; AF: areia fina; AT: areia total; Sil: silte; AR: argila; Md: mediana; s: desvio-padrão; Min.: valor mínimo; Max.: valor máximo; CV: coeficiente de variação; $\mathrm{Cs}$; coeficiente de assimetria; $\mathrm{C}_{\mathrm{K}}$ : coeficiente de curtose; $\mathrm{DN}$ : distribuição normal; ns: não significativo a 5\% pelo teste de Kolmogorov-Smirnov (KS), portanto, distribuição normal dos dados e *: distribuição não normal.

coeficientes de curtose, que é o grau de achatamento da curva de distribuição, todas as frações e o MC apresentaram distribuição platicúrtica, a exceção foi o silte que apresentou distribuição leptocúrtica. Pelo teste Kolmogorov-Smirnov as frações do solo e o MC apresentaram distribuição normal, o que pode ser verificado pela proximidade entre a média e mediana dos dados, já o silte não apresentou distribuição normal.

Ao analisar os coeficientes de variação (CV), segundo a classificação proposta por Warrick e Nielsen (1980), temos que: (AG) areia grossa, (AT) areia total e (MC) massa do cacho apresentaram um CV baixo $(<12 \%)$; as frações areia fina $(\mathrm{AF}) \mathrm{e}$ argila (AR) com média variação $(12 \%<\mathrm{CV}<60 \%)$ e alta variação (CV>60\%) com CV de $69 \%$ o silte (Sil), que pode estar relacionado com a forma de sua determinação que pela diferença entre as frações de areia e argila.

Os resultados observados para a normalidade dos dados concordam com os obtidos Lima (2007)

Tabela 2. Coeficiente de correlação de Pearson entre as frações granulométricas e a massa do cacho.

\begin{tabular}{lcrrrrr}
\hline Atributos & MC & AG & AF & AT & Sil & AR \\
\hline MC & \multirow{2}{*}{1,00} & $-0,02$ & $-0,04$ & $-0,07$ & $-0,05$ & 0,04 \\
AG & & 1,00 & $-0,66^{*}$ & $0,78^{*}$ & 0,11 & $-0,03$ \\
AF & & & 1,00 & $-0,04$ & $-0,16$ & $-0,03$ \\
AT & & & & 1,00 & 0,02 & $-0,74^{*}$ \\
Sil & & & & & 1,00 & $-0,10$ \\
AR & & & & & & 1,00 \\
\hline
\end{tabular}

*significativo pelo teste t ao nível de $5 \%$ de probabilidade; MC: massa do cacho; AG: areia grossa; AF: areia fina; AT: areia total; Sil: silte; AR: argila em solos cultivados com pimenta-do-reino, onde todas as frações apresentaram distribuição normal. Eguchi (2001) diz que se a distribuição não é normal, a média aritmética é uma medida muito influenciada por valores extremos, tornando-se uma medida de tendência central, não representativa do conjunto de dados. Porém, a normalidade dos dados não é uma exigência da geoestatística.

Análise de correlação linear de Pearson entre as frações granulométricas do solo e a produção da bananeira está apresentada na Tabela 2. Observa-se que o MC não apresentou correlação significativa com nenhuma das frações granulométricas. Isso demonstra que as frações granulométricas não influenciaram a produção da bananeira no primeiro ciclo, mas um estudo mais detalhado em longo prazo nos ciclos sucessivos poderá demonstrar uma possível correlação, ou ratificar o ocorrido no primeiro ciclo.

Os parâmetros e modelos ajustados para os semivariogramas estão apresentados na Tabela 3. O Sil apresentou ausência de dependência espacial (EPP) para distâncias maiores que a menor utilizada na amostragem, mostrando que as amostras são independentes. Isso indica que, para esse atributo a diferença entre os valores das amostras acontecem por acaso, não mostrando dependência espacial, independentemente do sistema de cultivo, podendo usar o valor da média para representar esse atributo. As demais frações granulométricas e a MC apresentaram dependência espacial com ajuste do modelo esférico (ESF), concordando aos dados de Vieira (1997) e Bertolani (2001), onde afirmam que o esférico é o modelo de maior ocorrência para os atributos do solo. 
Tabela 3. Resultados dos modelos ajustados e parâmetros dos semivariogramas para as frações granulométricas do solo e produção.

\begin{tabular}{|c|c|c|c|c|c|c|c|c|}
\hline \multirow[b]{2}{*}{ Atributos } & \multirow[b]{2}{*}{ Modelo } & \multirow[b]{2}{*}{$\mathrm{a}(\mathrm{m})$} & \multirow[b]{2}{*}{$\mathrm{C}_{0}$} & \multirow[b]{2}{*}{$\mathrm{C}_{0}+\mathrm{C}$} & \multirow[b]{2}{*}{$\operatorname{IDE}(\%)$} & \multirow[b]{2}{*}{$\mathrm{R}^{2}(\%)$} & \multicolumn{2}{|c|}{ Validação cruzada } \\
\hline & & & & & & & $\mathrm{r}$ & p-valor \\
\hline MC & EXP & 11 & 0,16 & 0,95 & 95 & 59 & 37 & 0,000 \\
\hline AG & ESF & 14,0 & 0,14 & 1,07 & 87 & 84 & 25 & 0,000 \\
\hline $\mathrm{AF}$ & ESF & 8,5 & 0,15 & 0,96 & 85 & 51 & 05 & 0,042 \\
\hline AT & ESF & 17,6 & 0,31 & 1,08 & 71 & 73 & 08 & 0,008 \\
\hline Sil & EPP & - & 1,0 & 1,0 & - & - & - & - \\
\hline AR & ESF & 9,5 & 0,21 & 1,03 & 80 & 63 & 03 & 0,563 \\
\hline
\end{tabular}

MC: massa do cacho; AG: areia grossa; AF: areia fina; AT: areia total; Sil: silte; AR: argila; ESF: modelo esférico; EPP: efeito pepita puro; a: alcance; $\mathrm{C}_{0}$ : efeito pepita; $\mathrm{C}_{0}+\mathrm{C}$ : patamar; IDE: índice de dependência espacial $\left(\mathrm{C} / \mathrm{C}_{0}+\mathrm{C}\right) ; \mathrm{R}^{2}$ : coeficiente de determinação do ajuste; r: coeficiente de correlação da validação cruzada e p-valor: nível de significância do valor observado pelo valor estimado pela validação cruzada.

Os alcances de dependência espacial para as frações AT e AG são de 14,0 e 17,6 m, respectivamente, indicando padrão espacial próximo e que a AG contribui mais na AT do que a AF. A argila (AR), areia fina (AF), e a massa do cacho (MC), apresentaram também, o mesmo padrão espacial com alcances de 9,5, 8,5 e $11 \mathrm{~m}$, respectivamente. Corá et al. (2004) afirmam que valores de alcance influenciam na qualidade das estimativas, uma vez que ele determina o número de valores usados na interpolação, assim estimativas feitas com interpolação por krigagem ordinária utilizando valores de alcances maiores tendem a ser mais confiáveis, apresentando mapas que representam melhor a realidade.

$\mathrm{O}$ índice de dependência espacial (IDE) apresentou forte para as frações AG, AF, AR e MC e moderada para a AT. O Coeficiente de determinação múltipla $\mathrm{R}^{2}$ variou de $51 \%$ (AF) a $84 \%$ (AG), que segundo Azevedo (2004) quando $\mathrm{R}^{2}$ for acima de $50 \%$, melhor será a estimativa de valores pelo método de interpolação krigagem ordinária.

Para os atributos que apresentaram dependência espacial, construíram-se mapas de isolinhas por krigagem ordinária com intuito de estimar valores em locais não medidos (Figura 1). Observa-se nos mapas que as frações AT e AG apresentam distribuição espacial com certa similaridade, mostrando uma alta correlação entre esses atributos, com maior concentração de sul para norte, apesar de correlação baixa com esta direção. Em função dos alcances encontrados, como era de se esperar, a distribuição de AR e de AF, também apresentam similaridade na área.
$\mathrm{Na}$ área em estudo, verifica-se a presença de zonas com maior concentração de argila (AR), isso se deve ao fato da existência, na época da implantação da cultura, de uma microforma no relevo denominada de murundus, que foi aplainado no processo de preparo do solo.

Verifica-se que o estudo da variabilidade espacial das frações granulométricas do solo são de extrema importância, visto que a fertilidade do solo (absorção de nutrientes), retenção de água e condições físicas favoráveis ao desenvolvimento do sistema radicular das plantas estão diretamente relacionado com a distribuição das frações constituintes, mas no presente estudo observou-se que não influenciaram a produção da bananeira.

Cabe ressaltar que as alterações nas frações granulométricas do solo provenientes do escoamento superficial são possíveis, mesmo tratando-se de áreas planas como do presente trabalho. Portanto a variabilidade espacial apresentada pelas frações granulométricas está relacionada aos fatores de formação do solo, já que dificilmente o manejo do solo alteraria a composição granulométrica, tampouco promoveria seu arranjamento espacial, conforme discutido por Berner et al. (2007).

A Figura 2 mostra a distribuição espacial da massa do cacho (MC), sendo o valor médio encontrado de 11,05 kh/planta, localizado entre os valores de 9,5 a $12 \mathrm{~kg} /$ planta na escala adotada, representando grande extensão da área. Observase ainda, a não existência de similaridade com a distribuição espacial das frações granulométricas, o que é comprovado pela ausência de correlação entre eles. 

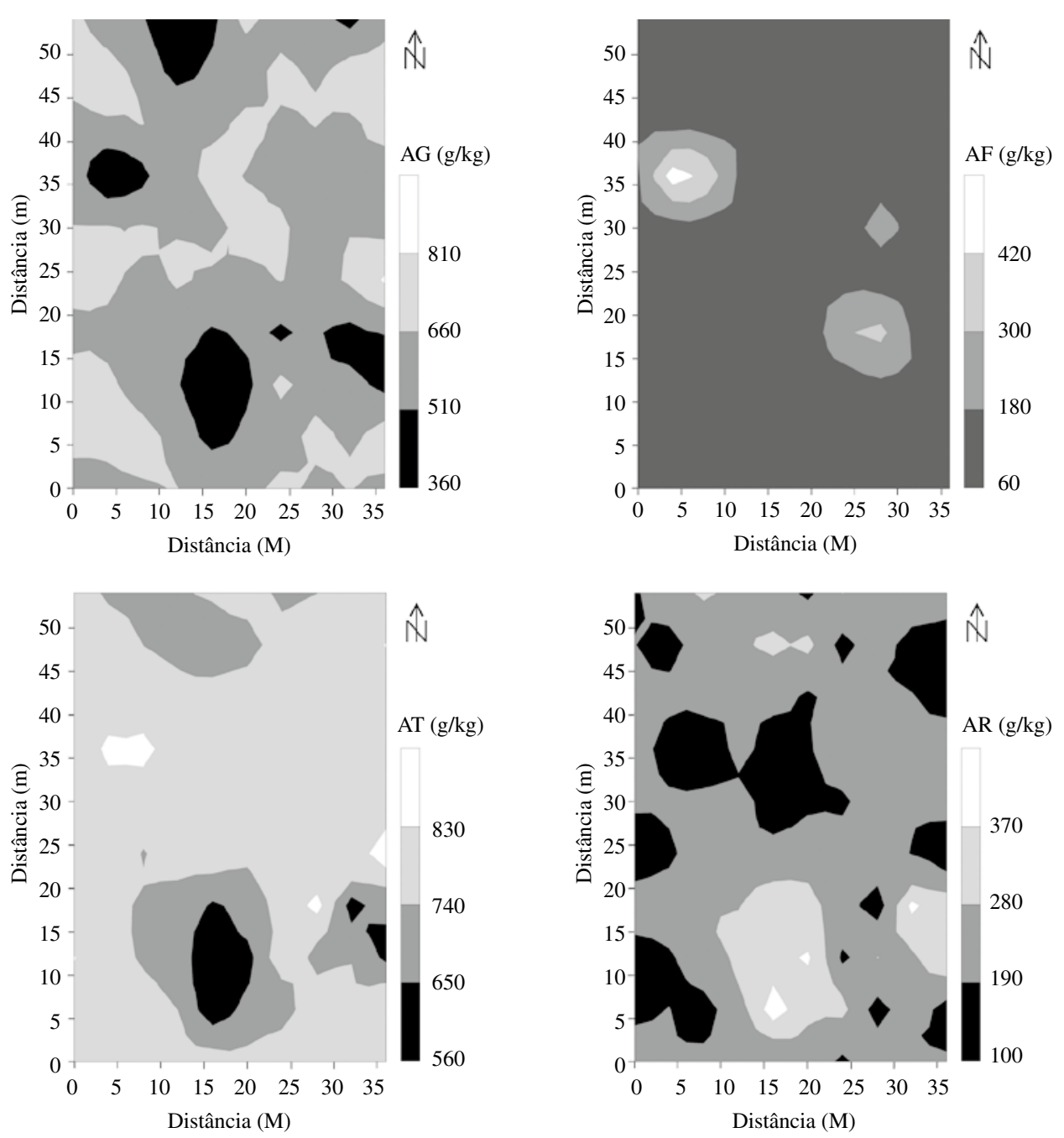

Figura 1. Mapas de isolinhas das frações do solo, areia grossa (AG), areia fina (AF), areia total (AT) e argila (AR).

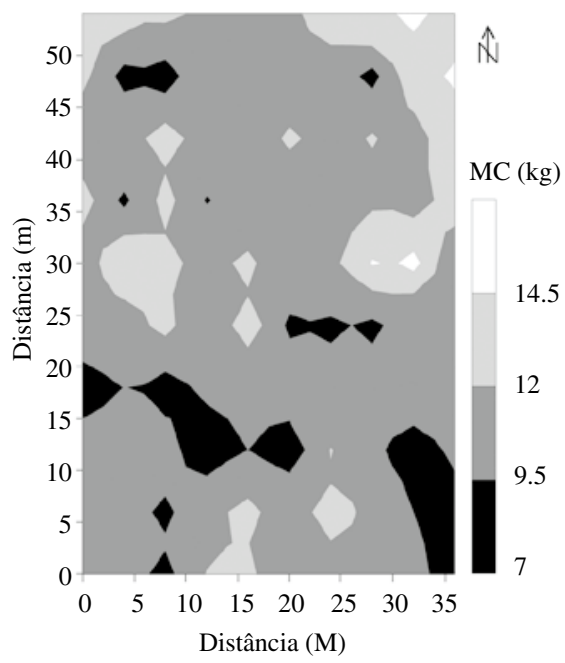

\section{Conclusões}

Nas condições em que foi realizado este experimento, pode-se concluir que:

- A massa do cacho (MC) não apresentou correlação significativa com nenhuma das frações granulométricas.

- Os atributos areia grossa (AG), areia fina (AF), areia total (AT), argila (AR) e (MC) massa do cacho apresentaram dependência espacial.

Figura 2. Mapa de isolinhas da distribuição espacial da produção de bananeira (massa do cacho por planta-kg/planta). 


\section{Referências Citadas}

Azevedo, E.C.

2004 Uso da geoestatística e de recursos de geoprocessamento no diagnóstico da degradação de um solo argiloso sob pastagem no estado de Mato Grosso. Campinas: 2004. 141 f. Tese (Doutorado em Engenharia na Agricultura) UNICAMP.

Berner, P.G.M.; Vieira, S.R.; Lima, E.; Anjos, L.H.C.

2007 Variabilidade espacial de propriedades físicas e químicas de um Cambissolo sob dois sistemas de manejos de canade-açucar. Viçosa. Revista Brasileira de Ciências do Solo. 31: $387-844$

Bertolani, F.C.; Vieira, S.R.

2001 Variabilidade espacial da taxa de infiltração de água e da espessura do horizonte A, em um Argissolo VermelhoAmarelo, sob diferentes usos. Revista Brasileira de Ciências do Solo, Viçosa, 25 (4): 987-995.

Cordeiro, Z.J.M.

2000 Aspectos gerais da Bananicultura. Brasília: Fundação Cargill, pp. 28-29.

Corá, J.E.; Araujo, A.V.; Pereira, G.T.; Beraldo, J.M.G.

2004 Variabilidade espacial de atributos do solo para adoção do sistema de agricultura de precisão na cultura de cana-de-açúcar. Revista Brasileira de Ciência do Solo, 28 (6): 1013-1021.

Duarte, M.N.; Curi, N.; Pérez, D.V.; Kampf, N.; Claessem, M.E.C.

2000 Minerologia, Química e Micromorfologia de solos de uma bacia nos tabuleiros costeiros do Espírito Santo. Pesquisa Agropecuária Brasileira, Brasília, 35 (6): 1237-1250.
Embrapa

1997 Centro Nacional de Pesquisa de Solos. Manual de métodos de análise de solo. 2. ed. Rio de Janeiro, 212 p.

Eguchi, E.S.; Silva, E.L.; Oliveira, M.S.

2002 Variabilidade especial da textura e da densidade de partículas em um solo Aluvial no Município de Lavras-MG. Revista Brasileira Engenharia Agrícola Ambiental, 9 (2): 242-246.

Lima, J.S. de S.; Oliveira, R.B.; Quartezani, W.Z.

2007 Variabilidade Espacial de Atributos Físicos de um Latossolo Vermelho-Amarelo sob cultivo de Pimenta-do-Reino. Engenharia na Agricultura, Viçosa, MG, 15 (3): 290-298.

Robertson, G.P.

$1998 \mathrm{GS}^{+}$: Geostatistics for the environmental sciences-GS ${ }^{+}$ User's Guide. Plainwell, Gamma Design Software, 152 p.

Souza, L.S.

1992 Variabilidade espacial do solo em sistemas de manejo. 162f. Tese (Doutorado) UFRGS, Porto Alegre, 1992.

Vieira, S.R.

1997 Variabilidade espacial de argila, silte e atributos químicos em uma parcela experimental de um Latossolo roxo de Campinas. Bragantia, Campinas, 56 (1): 1-13.

Warrick, A.W.; Nielsen, D.R.

1980 Spatial variability of soil physical properties in the field. In: Hillel, D. (Ed.). Applications of soil physics. New York: Academic, pp. 319-344.

Zimback, C.R.L.

2001 Análise especial de atributos químicos de solos para fins de mapeamento da fertilidade. Botucatu: 2001. $114 \mathrm{f}$. Tese (Doutorado em Energia na Agricultura) UNESP. 